\title{
Procedural factors associated with early postoperative arrhythmias after repair of congenital heart defects
}

\author{
Jean-Pierre Pfammatter, $\mathrm{MD}^{\mathrm{a}}$ \\ Bendicht Wagner, $\mathrm{MD}^{\mathrm{b}}$ \\ Pascal Berdat, MD \\ Denis C. G. Bachmann, MD \\ Mladen Pavlovic, MD \\ Jürg Pfenninger, $M D^{\mathrm{b}}$ \\ Thierry Carrel, MDc
}

Objectives: Early postoperative arrhythmias frequently are a relevant problem in the early postoperative management after surgical intervention for congenital heart disease. Few data are available indicating risk factors for their occurrence. The hypothesis was tested that factors closely related to the surgical procedure itself were associated with a higher incidence of arrhythmias early in the postoperative course after repair of congenital heart disease.

Methods: All consecutive patients undergoing 1 of 3 well-defined surgical procedures were prospectively evaluated for the occurrence of arrhythmias during the entire postoperative hospital stay by means of continuous electrocardiographic monitoring in the intensive care unit and use of 24-hour Holter monitors. Patients examined were those undergoing transatrial closure of a ventricular septal defect, repair of complete atrioventricular canal, and tetralogy of Fallot. The relation between procedural variables and the occurrence of arrhythmias was independently evaluated for each of these 3 heart defects.

Results: Early postoperative arrhythmias occurred in $30 \%$ of patients with ventricular septal defect $(n=75), 35 \%$ of patients with tetralogy of Fallot $(n=52)$, and $47 \%$ of patients with atrioventricular canal $(n=45)$. Patients with arrhythmias tended to be younger (significant only in the ventricular septal defect group). In all 3 patient groups, there was a significant correlation between incidence of arrhythmias and longer extracorporeal bypass time $(P<.05)$ and longer aortic crossclamp time $(P<.01)$, as well as with higher maximum postoperative troponin serum levels $(P<.01)$. In patients with atrioventricular canal, there was a significant relation between hemodynamically incomplete surgical results and the occurrence of From the Divisions of Pediatric Cardiology, Pediatric Intensive Care, ${ }^{\mathrm{b}}$ and Cardiovascular Surgery, ${ }^{c}$ University Hospital, Berne, Switzerland.

Received for publication May 4, 2001; revisions requested June 11, 2001; revisions received June 22, 2001; accepted for publication Aug 3, 2001.

Address for reprints: J. P. Pfammatter, MD, University Children's Hospital, Freiburgstrasse, CH 3010 Berne, Switzerland (E-mail: Jean.Pierre.Pfammatter@insel.ch).

J Thorac Cardiovasc Surg 2002;123:258-62

Copyright (†) 2002 by The American Association for Thoracic Surgery

0022-5223/2002 $\$ 35.00+0 \quad \mathbf{1 2 / 1 / 1 1 9 7 0 1}$

doi:10.1067/mtc.2002.119701 arrhythmias $(P<.01)$.

Conclusions: The occurrence of early postoperative arrhythmias after repair of congenital heart disease was significantly associated with procedure-related risk factors in each of 3 independent patient groups undergoing well-defined surgical procedures.

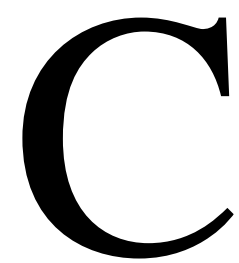

ardiac arrhythmias are a frequent problem in the early postoperative course after cardiac surgery. The question of risk factors predisposing to the occurrence of early postoperative arrhythmias has been addressed in adults ${ }^{1}$ but not systematically in an immediate postoperative pediatric population.

The aim of the present study was to assess the influence of perioperative procedure-related factors on the occurrence of arrhythmias during the immediate postoperative stay in the intensive care unit. These procedure-related factors were assessed 
independently in 3 groups of pediatric patients with identical surgical approaches, namely in children undergoing transatrial repair of ventricular septal defect (VSD), repair for tetralogy of Fallot (TOF), and surgical correction of complete atrioventricular septal defect (CAVC).

\section{Methods \\ Patients}

Beginning in July 1996, we prospectively monitored the occurrence of arrhythmias in the early postoperative course of all patients undergoing 1 of 3 well-defined surgical procedures for repair of congenital heart disease: transatrial patch closure of a VSD, total correction of TOF, and total correction of CAVC. For better comparison of risk factors for early postoperative arrhythmias, only standard surgical procedures were evaluated, and patients were excluded if the surgical procedure significantly differed from the standard approach or if, intraoperatively, the surgeon had to deal with unanticipated difficulties (eg, need for ventriculotomy in VSD repair and mitral valve replacement in CAVC repair).

The study was approved by the institution's ethical committee.

\section{Assessment of Procedure-related Factors}

The following variables were evaluated with regard to a possible relationship with the occurrence of early postoperative arrhythmias: the total duration of extracorporeal circulation (ECC) and the aortic crossclamp (ACC) time. Cutoff values for these parameters were used on the basis of values evaluated during an earlier study and allowing for adequate sample sizes in the subgroups. The maximum postoperative serum troponin I (TnI) level (measured routinely on arrival in the intensive care unit and then at 8, 24, and 48 hours postoperatively by means of immunoassay) and the patient's age at the time of the operation were recorded. The hemodynamic result of the repair was also assessed, with the patients assigned to either the group of patients with hemodynamically complete repair or the groups of patients with one or more of the following hemodynamic residual findings: significant residual left-right shunting ( $\geq 3 \mathrm{~mm}$ color-Doppler jet at ventricular level), residual pulmonary stenosis in patients with TOF (peak Doppler gradient $>30 \mathrm{~mm} \mathrm{Hg}$ ), persistent pulmonary hypertension ( $>50 \%$ of systemic pressure, as measured routinely with an intraoperatively placed pulmonary artery catheter) in patients with postoperative CAVC, and more than moderate valvular regurgitation (aortic valves in VSD repair, pulmonary valves in TOF repair, and atrioventricular valves in CAVC). In TOF preoperative cyanosis was also analyzed as a variable with saturation cutoff points for grouping of patients set at less than $80 \%, 80 \%$ to $89 \%$, and greater than $90 \%$.

The operations were performed under conditions of moderate hypothermia $\left(28^{\circ} \mathrm{C}-32^{\circ} \mathrm{C}\right)$, with cold-blood antegrade cardioplegia for myocardial protection. At the end of the operation, temporary epicardial wire leads were placed in routine fashion on the right atrium and ventricle in all patients. The same 2 surgeons (P.B. and T.C.) were involved in all operations throughout the study. Only surgical procedures that were electively planned and performed in stable patients were included in the study, excluding emergency interventions with preexisting hemodynamic compromise. In patients with VSD, only those patients with transatrial patch closure were included; the standard procedure usually consisted of an anterolateral right atrial incision. Repair of TOF was performed through a right atrial incision and a short ventriculotomy in the right outflow tract. CAVC repair was always done through a right atriotomy only.

\section{Definitions and Assessment of Arrhythmias}

The following were considered to be significant arrhythmias when occurring at one time during the entire postoperative hospital stay: second- or third-degree atrioventricular block; sinoatrial nodal dysfunction (defined as sinus bradycardia and normofrequent junctional escape rhythm); accelerated junctional rhythm (defined as narrow complex tachycardia without atrioventricular dissociation); junctional ectopic tachycardia (defined as narrow complex tachycardia with atrioventricular dissociation); supraventricular tachycardia either as atrioventricular reentrant (retrograde p-waves or 1:1 atrioventricular conduction shown by connecting atrial wires to the electrocardiograph) or atrial flutter (narrow complex tachycardia with $>2: 1$ atrioventricular conduction or by detecting flutter waves connecting atrial wires to electrocardiography machine); and ventricular tachycardia (tachycardia with atrioventricular dissociation and with broad QRS complex or different from QRS seen in sinus rhythm). For atrial or ventricular ectopic beats, an arbitrary quantitative cutoff point was used: ectopic beats greater than $10 \%$ of QRS complexes were considered relevant, otherwise they were not listed among the arrhythmias. For the purpose of risk-factor analysis, each patient entered one category of arrhythmias.

The usual postoperative electrocardiographic monitoring consisted of a continuous 1-lead electrocardiogram displayed on an overhead monitor (Hewlett-Packard 54S; Hewlett-Packard Company, Palo Alto, Calif). This surveillance unit was connected to a central monitoring station with a memory function (NEC MultiSync LCD 1810; NEC, Santa Clara, Calif) where the same 1lead electrocardiogram was also displayed but where the last 12 minutes of the electrocardiogram were continuously saved in all cases. In addition, a heart-rate trend curve was always stored for the last 24 hours. In case of observation of arrhythmias, specific time frames of the patient's electrocardiogram were printed out for detailed analysis. The patients were usually continuously monitored in the intensive care unit until resolution of any relevant postoperative rhythm disturbances.

Before discharge, a standard 12-lead electrocardiogram was performed together with a 24-hour Holter monitor in case the patient had relevant postoperative arrhythmias.

\section{Statistics}

Descriptive values are shown as means \pm SD or medians with ranges where appropriate. Comparisons between groups were made with either the unpaired Student $t$ test or the Mann-Whitney $U$ test where appropriate. Proportions were compared with the $\chi^{2}$ test or the Fisher exact test where appropriate.

\section{Results}

Transatrial Closure of VSD

A total of 75 pediatric patients had transatrial VSD closure during the study period, and 20 (30\%) children had early postoperative arrhythmias. Arrhythmias consisted of sinoatrial nodal dysfunction with bradycardia (4 patients), junc- 
TABLE 1. Influence of patient's age and hemodynamic results of surgical intervention on incidence of postoperative arrhythmias

\begin{tabular}{lccc}
\hline & \multicolumn{2}{c}{ Patients } & \\
\cline { 2 - 3 } & With arrhythmia & No arrhythmia & P value \\
\hline Patients with VSD & & & \\
No. & 20 & 55 & \\
Mean age (y) & $1.7 \pm 2.0$ & $3.6 \pm 3.9$ & .04 \\
Incomplete result & $0 / 1$ & $1 / 1$ & NS \\
Patients with CAVC & & & \\
No. & 21 & 24 & \\
Mean age (y) & $0.9 \pm 2.1$ & $1.4 \pm 1.9$ & NS \\
Incomplete result & $9 / 11$ & $2 / 11$ & .01 \\
Patients with TOF & & & \\
No. & 18 & 32 & \\
Mean age (y) & $1.6 \pm 1.9$ & $2.3 \pm 2.6$ & NS \\
Incomplete result & $3 / 6$ & $3 / 6$ & NS \\
\hline NS, Not significant. & & &
\end{tabular}

TABLE 2. Relation between procedural factors and the occurrence of arrhythmias in patients with VSD $(n=75)$

\begin{tabular}{lcrc}
\hline & \multicolumn{2}{c}{ Patients with arrhythmia } & \\
\cline { 2 - 3 } & No. & $\%$ & Pvalue \\
\hline Maximum troponin level $(\mu \mathrm{g} / \mathrm{L})$ & & & \\
$<10$ & $8 / 50$ & 16 & \\
$10-50$ & $11 / 24$ & 46 & $<.01$ \\
$>50$ & $1 / 1$ & 100 & \\
ECC $($ min) & & & \\
$<50$ & $2 / 29$ & 7 & \\
$50-100$ & $18 / 46$ & 39 & $<.01$ \\
ACC $($ min) & & & \\
$<30$ & $2 / 37$ & 5 & \\
$30-60$ & $18 / 38$ & 47 & $<.01$ \\
\hline
\end{tabular}

tional tachycardia (3 patients), episodes of supraventricular tachycardia (3 patients), transient complete atrioventricular block (4 patients), and frequent supraventricular and ventricular ectopic beats ( 6 patients). Arrhythmias had ceased by 72 hours postoperatively (median, 24 hours) in all patients.

The hemodynamic result of repair was considered incomplete (residual shunt) in only 1 patient, who did not have arrhythmias. Patients who had arrhythmias were significantly younger (mean, $1.7 \pm 2$ vs $3.6 \pm 3.9$ years; $P=$ .04 ; Table 1) than those without arrhythmias.

As shown in Table 2, the proportion of children with postoperative arrhythmias showed a significant increase, from $16 \%$ in patients with maximum postoperative $\mathrm{TnI}$ levels of less than $10 \mu \mathrm{g} / \mathrm{L}$ to $46 \%$ when the maximum TnI level was in the range of 10 and $50 \mu \mathrm{g} / \mathrm{L}(P<.01)$. There
TABLE 3. Relation between procedural factors and the occurrence of arrhythmias in patients with TOF $(n=52)$

\begin{tabular}{lccc}
\hline & \multicolumn{2}{c}{ Patients with arrhythmia } & \\
\cline { 2 - 3 } & No. & $\%$ & P value \\
\hline Maximum troponin level $(\mu \mathrm{g} / \mathrm{L})$ & & & \\
$\quad<50$ & $10 / 41$ & 24 & \\
$50-100$ & $5 / 8$ & 63 & $<.01$ \\
$>100$ & $3 / 3$ & 100 & \\
ECC $($ min) & & & \\
$50-100$ & $9 / 35$ & 26 & \\
$>100$ & $9 / 17$ & 53 & $<.05$ \\
ACC $($ min) & & & \\
$<50$ & $3 / 28$ & 11 & \\
$50-100$ & $15 / 24$ & 63 & $<.01$ \\
\hline
\end{tabular}

was only 1 patient with TnI values exceeding $50 \mu \mathrm{g} / \mathrm{L}$, and he had arrhythmias. As a group, patients with arrhythmias had significantly higher TnI levels (mean, $21 \pm 26$ vs $9 \pm 8$ $\mu \mathrm{g} / \mathrm{L} ; P<.01)$.

The same pattern was found for prolonged ECC and ACC times. When the ECC time remained less than 50 minutes, $7 \%$ of patients had an arrhythmia, whereas this proportion rose to $39 \%$ when ECC was greater than 50 minutes $(P<$ $.01)$. For patients with an ACC time of less than 30 minutes, arrhythmias were observed in 5\%; for ACC times of between 30 and 60 minutes, this proportion was $47 \%(P<.01)$. As a group, children with arrhythmias had significantly longer ECC duration (mean, $68 \pm 17$ vs $53 \pm 14$ minutes; $P<.01$ ) and ACC times (mean, $42 \pm 15$ vs $31 \pm 9$ minutes; $P<.01$ ).

\section{Repair of TOF}

Of 52 patients with TOF operated on during the study period, $18(35 \%)$ had an arrhythmia in the early postoperative period. Arrhythmias observed were accelerated junctional rhythm (7 patients), junctional ectopic tachycardia (2 patients), sinus node dysfunction with bradycardia (2 patients), complete atrioventricular block (2 patients), supraventricular tachycardia (1 patient), and frequent ectopic beats (4 patients). These arrhythmias all disappeared by 48 hours postoperatively (median, 24 hours).

Only 6 patients had a hemodynamically incomplete surgical result, and 3 of these patients had arrhythmias; this small number did not allow for a statistical comparison with the total population. As a group, the patients who had arrhythmias tended to be younger (mean, $1.6 \pm 1.9$ vs $2.3 \pm$ 2.6 years; $P=.1$ ). The degree of preoperative cyanosis was not a predictor of postoperative arrhythmias. As a group, patients with arrhythmias had significantly higher maximum postoperative TnI levels (mean, $60 \pm 71$ vs $29 \pm 18$ $\mu / \mathrm{L} ; P=.02)$ and longer ACC times $(P<.01)$, whereas there was only a trend toward longer ECC times (Table 3 ). 
As shown in Table 3, the incidence of arrhythmias significantly increased when patients were grouped according to the maximum TnI levels, longer ECC times, and longer ACC times. The duration of postoperative arrhythmias did not correlate with either of these variables (data not shown).

\section{Surgical Correction of CAVC}

Among patients with CAVC, 36 of 45 had Down syndrome. Twenty-one $(47 \%)$ children had arrhythmias during the postoperative course. The arrhythmias encountered were accelerated junctional rhythm in 8 patients, sinus node dysfunction with bradycardia in 7 patients, complete atrioventricular block in 4 patients (permanent in 1 patient), atrial flutter in 1 patient, and junctional ectopic tachycardia in 1 patient. Three children had frequent ectopic beats also. Arrhythmias lasted for a maximum of 128 hours postoperatively (median, 22 hours).

Hemodynamically relevant residual postoperative findings were seen in 11 patients (persistent pulmonary hypertension in 3 , residual VSD in 2 , and mitral regurgitation in $6)$. In that group patients with arrhythmias were significantly overrepresented $(P<.01$, Table 1$)$. As in the other defects evaluated, patients with arrhythmias showed a trend toward being younger $(P=.1$, Table 1$)$. As was observed in patients after repair for VSD and TOF, a significant increase in the proportion of patients with postoperative arrhythmias was found to be associated also after CAVC repair with higher postoperative TnI values $(P<.01)$, longer ACC times $(P<.05)$, and longer duration of ECC $(P<.01$, Table 4$)$.

\section{Clinical Effect of Arrhythmias}

Of all 59 patients with arrhythmias, treatment was required in 37 , consisting of transient pacing in 32 , body surface cooling in 3, and/or antiarrhythmic drugs in 4 . No treatment was required in 22 patients. Intensive care unit stay was considered to be prolonged as a result of arrhythmias in 10 patients. Only 2 children had antiarrhythmic drug treatment at discharge.

\section{Discussion}

Arrhythmias occurring early in the postoperative course after repair of congenital heart disease are frequent and may become a relevant clinical problem. This was again documented in the present study, with arrhythmias occurring in $30 \%$ to $47 \%$ of such patients. Studies conducted so far on cardiac arrhythmias in the immediate postoperative period after surgery for congenital heart disease focused mainly on specific cardiac defects, ${ }^{2}$ specific arrhythmias, ${ }^{3}$ or specific treatment at that stage. ${ }^{4-8}$ Reports on arrhythmias in the long-term follow-up after cardiac surgery largely outnumber the few studies on immediate postoperative arrhythmias.

The main findings of our study were that procedurerelated factors were significantly related to the incidence of
TABLE 4. Relation between procedural factors and the occurrence of arrhythmias in patients with CAVC $(n=45)$

\begin{tabular}{lccc}
\hline & \multicolumn{2}{c}{ Patients with arrhythmia } & \\
\cline { 2 - 3 } & No. & $\%$ & P value \\
\hline Maximum troponin level $(\mu \mathrm{g} / \mathrm{L})$ & & & \\
$\quad<10$ & $1 / 10$ & 10 & \\
$10-50$ & $11 / 25$ & 44 & $<.01$ \\
$50-100$ & $6 / 7$ & 86 & \\
$>100$ & $3 / 3$ & 100 & \\
ECC $(\min )$ & & & \\
$50-100$ & $15 / 36$ & 42 & \\
$>100$ & $6 / 9$ & 66 & $<.05$ \\
ACC $(\min )$ & & & \\
$<50$ & $2 / 18$ & 11 & \\
$50-100$ & $19 / 27$ & 70 & $<.01$ \\
\hline
\end{tabular}

rhythm disturbances early after corrective surgery for congenital heart defects. For all 3 types of surgical procedures examined, we found that increasing ECC duration and longer ACC time were strong predictors of postoperative arrhythmias. This evidence was hardly surprising, but this relationship has not been systematically evaluated thus far in specific patient groups with surgical repair for the same congenital heart defect done by the same surgeons. An interesting and new finding of the present study was that the incidence of immediate postoperative arrhythmias also correlated with the maximum postoperative serum levels of cardiac troponin. As we compared the occurrence of arrhythmias in 3 groups of patients with comparable surgical procedures, we were able to exclude as far as possible factors associated with differences in the technical approach, in severity of underlying heart defects, or in the hemodynamic condition within the heterogeneous field of pediatric cardiac surgical patients.

The uniform pattern of predisposing factors for early postoperative arrhythmias in all 3 groups of patients allows for a common hypothesis with regard to the development of arrhythmias. The risk factors found seemed to be independent of the surgical access to the heart (either ventriculotomy or atriotomy). It has already been shown for patients with VSD that, in the long run, the site of surgical access (atriotomy vs ventriculotomy) had no true relevance for the occurrence of arrhythmias late after surgical intervention. ${ }^{9}$

The procedure time-related risk factors that were found may stand for a diffuse and unspecific damage to the myocardium that also translated into higher maximum postoperative serum levels of cardiac TnI, as has already been shown by our group. ${ }^{10,11}$ Whereas surgically induced local myocardial damage can be assumed to be the causative factor in the occurrence of complete postoperative block, local surgical damage has also been shown to be probably 
causative for another rare type of self-limiting arrhythmia typically occurring in the early postoperative setting, namely junctional ectopic tachycardia. ${ }^{12}$ The results of our current study might indicate that, more likely than such local factors, diffuse myocardial damage, inevitably induced by periprocedural tissue hypoxia and ischemia, created the substrate for the majority of arrhythmias in the early postoperative course. This would also be in contrast to the supposed cause of the majority of late postoperative arrhythmias, which were demonstrated to originate mainly around surgical scars. ${ }^{13,14}$

In the group of patients who had CAVC repair, it was moreover shown that hemodynamically relevant residual findings caused by technically incomplete repair, translating into pressure or volume overload of one cardiac chamber, might predispose to arrhythmias (patients with CAVC were the only of the 3 groups in which such residual findings were frequent enough to allow for statistical evaluation). This observation to our knowledge has not been reported before. Thus, in that vulnerable period of recovery from intraoperative arrest, hemodynamic disturbances otherwise well tolerated outside that immediate perioperative period might predispose, by additional diffuse myocardial stress, to the occurrence of arrhythmias.

Only very few data are available with regard to the effect and predisposing factors for arrhythmias early after pediatric cardiac surgery. To our knowledge, only one study thus far addressed the question of risk factors for early postoperative arrhythmias in the pediatric age group. That study was conducted in a more heterogeneous and mixed pediatric intensive care population, but similar findings to ours have been reported. ${ }^{15}$ The overall incidence of postoperative arrhythmias was $43 \%$ and thus comparable with the incidence found in our population. According to these results, longer cardiopulmonary bypass time predisposed to certain types of arrhythmias (ventricular tachycardia and junctional ectopic tachycardia). No correlation with postoperative TnI values was done nor were the arrhythmias correlated with postoperative hemodynamics.

The current study allowed for the description of some procedure-related factors that were associated with an increased incidence of rhythm disturbances in the early postoperative course. The major limitation of the present evaluation was that by far not all variables occurring during or early after pediatric cardiac surgery could be assessed. There are inherent individual differences that could not be addressed, even in operations for the same defect done by the same surgeon, nor was it possible to take into account postoperative variations of electrolytes or inotropic support and evaluate their effects on arrhythmias.

\section{References}

1. Chung MK. Cardiac surgery: postoperative arrhythmias. Crit Care Med. 2000;28(suppl):N136-43.

2. Kurer CC, Tanner CS, Norwood WI, Vetter VL. Perioperative arrhythmias after Fontan repair. Circulation. 1990;82(Suppl):IV-190-4.

3. Weindling SN, Saul PJ, Gamble WJ, Mayer JE, Wessel D, Walsh EP. Duration of complete atrioventricular block after congenital heart disease surgery. Am J Cardiol. 1998;82:525-7.

4. Janousek J, Vojtovic V, Chaloupecky V, Hucin B, Tlaskal T, Kostelka $\mathrm{M}$, et al. Hemodynamically optimized temporary cardiac pacing after surgery for congenital heart defects. Pacing Clin Electrophysiol. 2000;23:1250-9.

5. Rossi AF, Steinberg LG, Kipel G, Golinko RJ, Griepp RB. Use of adenosine in the management of perioperative arrhythmias in the pediatric cardiac intensive care unit. Crit Care Med. 1992;20:1107-11.

6. Humes RA, Porter CJ, Puga FJ, Schaff HV, Danielson GK. Utility of temporary atrial epicardial electrodes in postoperative pediatric cardiac patients. Mayo Clin Proc. 1989;64:516-21.

7. Pfammatter JP, Paul T, Ziemer G, Kallfelz HC. Successful management of junctional tachycardia by hypothermia after cardiac operations in infants. Ann Thorac Surg. 1995;60:556-60.

8. Walsh EP, Saul P, Sholler GF, Triedman JK, Jonas RA, Mayer JE, et al. Evaluation of a staged treatment protocol for rapid automatic junctional tachycardia after operation for congenital heart disease. $J \mathrm{Am}$ Coll Cardiol. 1997;29:1046-53.

9. Houyel L, Vaksman G, Fournier A, Davignon A. Ventricular arrhythmias after correction of ventricular septal defect: importance of surgical approach. J Am Coll Cardiol. 1990;16:1224-8.

10. Immer FF, Stocker F, Seiler AM, Pfammatter JP, Printzen G, Peheim E. Troponin T: improved diagnostic assessment of myocardial damage in childhood. Acta Paediatr. 1997;86:1321-7.

11. Immer FF, Stocker F, Seiler AM, Pfammatter JP, Bachmann D, Carrel T. Troponin-I for the prediction of early postoperative course after pediatric cardiac surgery. J Am Coll Cardiol. 1999;33:1719-23.

12. Till JA, Siew YH, Rowland E. Histopathological findings in three children with His bundle tachycardia occurring subsequent to cardiac surgery. Eur Heart J. 1992;13:709-12.

13. Horton RP, Canby RC, Kessler DJ, Joglar JA, Hume A, Jessen ME, et al. Ablation of ventricular tachycardia associated with tetralogy of Fallot. J Cardiovasc Electrophysiol. 1997;8:432-5.

14. Triedman JK, Bergau DM, Saul P, Epstein MR, Walsh EP. Efficacy of radiofrequency ablation for control of intraatrial reentrant tachycardia in patients with congenital heart disease. J Am Coll Cardiol. 1997;30: 1032-8.

15. Hoffman TM, Wernovsky G, Wieand TS, et al. Predictors of arrhythmias in a pediatric cardiac intensive care unit [abstract]. $J$ Am Coll Cardiol. 1999;34(suppl):539A. 